\title{
0661 MULTIPLE INJURY PROFILES OF DIFFERENT ROAD USERS IN NEW ZEALAND, 2000-2007
}

S Tin Tin*, A Woodward, S Ameratunga Correspondence: Section of Epidemiology and Biostatistics, School of Population Health, University of Auckland, Tamaki Campus, University of Auckland, Private Bag 92019, Auckland 1142, New Zealand

\subsection{6/ip.2010.029215.661}

Aim To compare profiles of traffic injuries sustained by different road users that resulted in death or hospital inpatient treatment.

Methods Traffic injuries were identified from the Mortality Collection and the National Minimum Dataset. The hospitalised sample was restricted to patients admitted to public hospitals with (a) a principal diagnosis of injury only, (b) hospital stay of one day or more and (c) first admissions only. Using a modified Barell matrix, the International Classification of Diseases injury diagnosis codes were classified into seven body regions and six injury types/natures. The 'multiple injury profiles' approach was used and up to ten diagnoses per case were extracted.

Results During 2000-2007, 13792 car/van drivers, 9252 car/ van passengers, 5881 motorcyclists, 5266 pedal cyclists and 4152 pedestrians were killed or admitted to hospital. The most common injuries (experienced by $>20 \%$ of specific road users injured) include:? Traumatic brain injuries - internal: occurred in $30.1 \%$ of pedestrians, $26.2 \%$ of drivers, $24.1 \%$ of passengers and $20.8 \%$ of pedal cyclists? Other head, face, neck - open wounds: occurred in $31.7 \%$ of drivers, $29.2 \%$ of passengers and $26.7 \%$ of pedestrians? Lower extremity - fracture: occurred in $38.8 \%$ of pedestrians, $35.0 \%$ of motorcyclists and $23.4 \%$ of drivers? Upper extremity - fracture: occurred in $34.4 \%$ of pedal cyclists and $31.1 \%$ of motorcyclists. The detailed matrix will be presented graphically for each road user.

Conclusion The injury profiles varied by mode of travel. This could help inform the prioritisation of future injury prevention, road safety and healthcare policies and interventions targeted at specific road users. 